\title{
MANEJO SUSTENTABLE DEL BOSQUE, DESDE LA COSMOVISIÓN MISKITU
}

Yader Galo-Sacasa ${ }^{[1]}$

Sandra Davis Rodríguez ${ }^{[2]}$

\section{Resumen}

El estudio se realizó en la comunidad de Miguel Bikan, territorio de Wanki Twi-Tasba Raya, Municipio de Waspam, Región Autónoma Atlántico Norte, Nicaragua; con el fin de caracterizar el manejo del bosque de la comunidad. Para dar repuesta al tema, se realizaron grupos focales y entrevista con informantes claves de la comunidad. La percepción de la gente de las comunidades hacia los ecosistemas bosque es que ella es la madre proveedora de todo lo necesario, de donde obtienen recursos y bienes intangibles necesarios para su existencia. Por lo tanto el aprovechamiento sustentable se hace de dos maneras: por un lado el aprovechamiento familiar, el cual se realiza en la parcela productiva de la familia y el aprovechamiento colectivo, que se realiza en el área de reserva comunal.

Palabras Clave: Sustentabilidad; Manejo del Bosque; comunidad.

\section{Summary}

The study was conducted in the community of Miguel Bikan, from the Wanki Twi Tasba Raya territory, municipality of Waspam, North Atlantic Autonomous Region of Nicaragua, with the purpose to characterize the community forest management. To be able to answer the topic, focus groups and interviews were carry-out with key informants from the community. The perception of the people in the communities concerning forest ecosystems is that she is the mother provider of everything that is needed, from where they obtain the necessary resources and intangible assets for their existence. Therefore, the sustainable use is made in two ways: first, the family use which is made in the family productive plot and second, the collective use which is done in the area of communal reserve.

Keywords: Sustainability; forest management; community.

[1] MSc. URACCAN, Bilwi. Bo El Muelle de FADCANIC 75 vrs al sur. nelsongalosacasa@gmail.com

[2] MSc. Coordinadora NITLAPAN-UCA. Bilwi, Costado Sur del estadio Municipal de Baseboll. diriangenus@yahoo.com 


\section{Introducción}

Los pueblos indígenas de la región centroamericana se han caracterizado por manejar los recursos naturales que se encuentran en sus territorios en base a normas de uso local y tradicional, bajo el principio de sostenibilidad. Lo anterior, en el caso de Nicaragua, se evidencia en los resultados del inventario nacional forestal (INAFOR, 2009), que refleja que $62.7 \%$ de la cobertura total nacional se encuentra en las Regiones Autónomas del Atlántico Norte. Por otro lado, el $87 \%$ de la cobertura boscosa de la RAAN se encuentra distribuida entre los 23 territorios indígenas definidos.

La comunidad indígena miskitu de Miguel Bikan tiene como recurso básico y de mayor importancia los bosques de pinares y latifoliadas. Ha sido una comunidad que históricamente ha subsistido de la agricultura, pesca y caza, lo cual está directamente relacionado con la sustentabilidad del bosque. En la actualidad, mantiene similares formas de vida comunal, con la variación de que han incluido algunas normativas que el Estado ha implementado a través del INAFOR de uso y regulación del recurso bosque. Esta forma de conservación orienta a la sustentabilidad del bosque basado en sus costumbres, tradiciones y cosmovisión, que ha conllevado a creación de normas de uso, acceso y control de determinados recursos y/o espacios naturales.

\section{Revisión de literatura}

\section{Cosmovisión}

La cosmovisión de un pueblo tiene relación directa con la naturaleza y con el uso de los recursos. Generalmente en un proceso de vida comunal de uso y aprovechamiento de recursos naturales para la subsistencia comunal, tiene su base en los significados simbólicos de la naturaleza, y esto de alguna forma regula las actividades. Esto es el principio de sostenibilidad de las comunidades indígenas, el utilizar lo justo para su subsistencia (Fondo Indígena, 2008:70).

Cox (1998:21 y 22), menciona que desde la cosmovisión miskita dentro de la relación con la naturaleza están los dioses:

- Wan Aisa o Wan Baikan uplika: Es el principio de la vida y de las cosas. Es el Dios Principal.

- Alwani: Dios del trueno.

- Ubia: Señor de las montañas y los árboles. 
- Liwa: Con sus mares, lagos y ríos. Todos ellos reúnen la espiritualidad, la religiosidad, la filosofía y hasta el valor cultural con sus temores, administraciones y sabiduría de nuestro pueblo.

- Niki-Niki: Semidios creador del río Wanki.

- Prahaku: Es el ser del tiempo y el viento, viaja en su eterna canoa de norte a sur. Cuando viaja de sur hacia norte, en la tierra se sufren las consecuencias.

El mismo autor menciona (1998:29), que en la religión miskitu los dioses no tienen cultos específicos. Los Sukia son los sacerdotes que han sobrevivido a la persecución de las iglesias foráneas.

Estos dioses y semidioses son los encargados de regular el uso y acceso a los recursos naturales, pues quien abuse o desobedezca a los mandatos de los dueños de los recursos puede ser castigado con alguna enfermedad o hasta con la muerte, si no es tratado por un sukia.

Por su parte, el Fondo Indígena (2008:71), relacionado a la cosmovisión de los pueblos indígenas, afirma que: "la forma de concebir, explicar y vivir la vida desde los pueblos indígenas; fundamenta los sistemas de vida, sistemas jurídicos y de organización político-social, así como el conjunto de valores, principios, creencias, normas y todas las formas de manifestaciones y expresiones propias de los pueblos indígenas".

En el mismo estudio, el Fondo Indígena menciona que:

la cosmovisión de los pueblos indígenas se basa en que el universo es un todo, es una integralidad, cada uno de los elementos que lo conforman tiene una razón de ser, están interrelacionados y son complementarios. De esta complementariedad surge el principio de equilibrio y armonía, así como el valor de la convivencia comunitaria, entendiendo lo comunitario, como el espacio y el tiempo en que interactúan todos los elementos del universo y no únicamente la convivencia entre humanos (2008:71).

Este planteamiento es la base para la sostenibilidad y sustentabilidad de los recursos naturales comunales.

Desde la cosmovisión maya, las montañas, los bosques, los cerros, los animales, el agua, los ríos, los mares, los lagos, las estrellas, los astros, la lluvia y todo su entorno, incluido los seres humanos, son parte de un todo por el que tienen vida. La Naturaleza es considerada la Madre Tierra, la Pachama, es el Jaw, la que los soporta, alimenta y protege (Pocop, 1992:39). 
De esta cita se puede interpretar que la tierra es considerada como una madre, porque de ella nacemos, en ella vivimos y de ella nos alimentamos. Por eso los pueblos indígenas asumen una relación espiritual con la tierra, se pide permiso al cortar un árbol, arrancar una piedra o perforar la tierra para sembrar el maíz.

FODIGUA (citado por Fondo Indígena, 2008:74), hace referencia a que la sostenibilidad de los recursos naturales comunitarios se centra en la armonía con las plantas y con los animales, el respeto al clima, la lluvia, los vientos y los montes.

La cosmovisión de los pueblos indígenas se basa en la relación armónica y holística en todos los elementos de la naturaleza al cual el ser humano pertenece pero no la domina. En la cosmovisión indígena no existe la lógica de un proceso lineal progresivo, sino más bien conceptos como la circularidad, el futuro que al mismo tiempo es pasado, el tiempo que se rige por los ciclos naturales del movimiento de los planetas y de los ciclos estacionales y agrícolas.

Deruyttere (2001), explica claramente el planteamiento anterior al decir:

su racionalidad económica no es de acumulación, sino de relación armónica con el entorno y uso respetuoso de los recursos naturales para el bienestar de toda la comunidad. Por lo tanto, en la economía indígena rigen los principios de reciprocidad y redistribución para que todos los miembros de la comunidad tengan acceso a los mismos niveles de bienestar.

Por su parte Danbolt (1997:27), menciona que la cosmovisión se utiliza para formar valores y normas, así mismo para explicar el propósito de la vida y el papel del ser humano en relación al universo, a los poderes sobrenaturales y en relación a los demás. Tiene una función explicativa sobre lo que uno encuentra en la vida.

En cuanto la formación de valores y normas, elementos fundamentales para optar a la sostenibilidad y sustentabilidad de los recursos naturales de uso comunal, Ostrom (1990), menciona que los valores tradicionales y normas son esenciales para la constitución de instituciones de uso colectivo. Para la autora, las reglas se refieren a las prescripciones compartidas, o de entendimiento mutuo entre las personas que se hacen cumplir en situaciones particulares mediante agentes encargados de monitorear e imponer sanciones.

La autora deja claro que es necesario el uso de normas en la organización de una comunidad para beneficio colectivo. La colectividad tiene fundamento en la distribución equitativa de los beneficios productivos y esto se relaciona mucho con la sostenibilidad de los recursos naturales manejados de manera colectica, como es el caso de territorios indígenas. 


\section{Metodología}

El estudio se llevó a cabo en la comunidad indígena Miguel Bikan, territorio de Tasba Raya $\left(28,224\right.$ hectáreas $\left.{ }^{[3]}\right)$, municipio de Waspam, cuenta con bosques latifoliados y de coníferas. Estos bosques han sido la base de subsistencia de la comunidad y a pesar de las presiones de agentes exógenos sobre el recurso, específicamente madera, los comunitarios han mantenido una relación armónica con el recurso bosque haciéndolo sustentable y sostenible en el tiempo, a través de normas de uso y acceso.

El estudio es cualitativo, bajo el paradigma crítico social y un enfoque de interaccionismo simbólico. El universo lo conforman 457 habitantes de la comunidad y la muestra fue realizada por saturación de información, tratando de involucrar a personas que trabajan directamente con los recursos naturales. Las técnicas utilizadas para la recopilación de la información fueron la entrevista semiestructuradas, grupo focal y pláticas informales.

Para la realización de este estudio, se obtuvo el consentimiento libre previo e informado de las autoridades comunales de Miguel Bikan, de manera verbal. El compromiso con los comunitarios es de regresar a la comunidad para presentar los resultados de la investigación para su aprobación y consentimiento para ser publicado.

Las entrevistas, los grupos focales y las pláticas informales se realizaron por petición de los informantes, en un cien por ciento en lengua materna, por lo que fue necesaria la ayuda de intérpretes miskitus y posteriormente un traductor y transcriptor de las grabaciones. Ambos aceptaron el compromiso de guardar secreto profesional de la información que estaban manejando y el nombre de los informantes.

\section{Resultados y discusión}

\section{El bosque de la comunidad de Miguel Bikan}

En el caso de la comunidad de Miguel Bikan el bosque de pinares se extiende al suroeste de la comunidad. El ecosistema se caracteriza por la presencia de la especie arbórea dominante, el pino del Caribe "Pinus caribaea var. hondurensis" (López, 2008a).

Según la descripción de López, (2008a), el área de bosque de pinares tiene una extensión de 490 ha. Se divide en rodales que se encuentran maduros y sobre maduro, con edades que oscilan entre los 27 y 50 años con áreas promedio de 40.7 has/rodal. Se estima que la producción total de madera es de unos $24,494.1 \mathrm{~m}^{3}$, lo que significan unos $60 \mathrm{~m}^{3} /$ ha.

[3] Según datos del Plan de desarrollo comunal elaborado por el CBA en el 2003. 
Entre los pinares se encuentran otras especies como el chaparro (Curatella americana L.), nancite (Byrsonima crassifolia), palma papta (Acoelorraphe wrightii) y roble sabanero (Tebebuia rosea), que son de gran importancia para la comunidad (leña, carbón, frutos). De igual manera, se encuentran especies faunísticas, como las loras verdes que anidan en los árboles podridos de pino, por lo que el ecosistema es clave para la sostenibilidad de la biodiversidad.

En cuanto al bosque de latifoliado, se estima que el área sean unas 800 ha (PANA PANA, 2009). En el año 2007 fue afectado en un $10 \%$ tras el paso del huracán Félix, sin embargo, ahora presenta estructuras normales en densidad y cobertura (PANA PANA, 2009).

López (2008b), estimó que un área de 60 has se pueden obtener unos $1,106.34 \mathrm{~m}^{3}$ de madera, lo que representa unos $18.44 \mathrm{~m}^{3} / \mathrm{h}$. Sin embargo, el volumen por hectárea, es relativamente bajo y por lo general en los bosques de latifoliado anda por los 168 $\mathrm{m}^{3} /$ ha por lo que es probable que estos datos de López (2008b) no sean representativo de todo el bosque de la comunidad de Miguel Bikan.

De igual manera, menciona que las especies maderables más representativas son: Caoba (Swietenia macrophylla), Guayabo negro (Terminalia amazonia), Níspero (Manilkara zapota) y Santa María (Calophyllum brasilliense) siendo ésta última la que mayores volúmenes por hectárea ha registrado con $14.56 \mathrm{~m}^{3} / \mathrm{ha}$.

Se observa la existencia de una base socioeconómica que representa en la población sus costumbres y los recursos forestales deseables, que podrían orientar medidas de sustentabilidad para la comunidad.

\section{Conocimiento del sistema bosque}

El conocimiento de las comunidades indígenas es un elemento que integra el uso de los recursos naturales, la cosmovisión y la espiritualidad. Son factores determinantes en la conformación del conocimiento de la población. En este sentido el Fondo Indígena (2008), hace referencia en que la "cosmovisión indígena está basada en que el universo es un todo, es una integralidad, cada uno de los elementos que lo conforma tienen una razón de ser, están interrelacionados y son complementarios".

Al consultar a los comunitarios de Miguel Bikan, sobre lo que es el bosque en su percepción, manifiestan:

El bosque es como una madre, allí nosotros sembramos, de allí sobrevivimos, de allí criamos a nuestros hijos, obtenemos todo de allí... por esos árboles es que respiramos aire puro, el viento que viene es muy limpio, esas son las cosas que obtenemos del 
bosque... un buen aire, si cortamos los árboles a las orillas de los ríos, estos se secan, pero si no pasa eso, el agua del río es helada y dulce.

Esta aseveración de los comunitarios nos explica que el bosque es como una madre para ellos, ya que le provee todo lo que necesita para sobrevivir, les provee espacio, alimento, agua y aire, de los cuales ellos dependen en todas las etapas de su vida. Pero también de la cita se puede rescatar que ellos son parte del ecosistema bosque. Es notable la estrecha relación de beneficio y respeto que existe entre los comunitarios y el bosque. De igual manera, el bosque no está limitado a recursos forestales como suele suceder en la cultura occidental y más bien se trata de un conjunto de recursos de los cuales pueden obtener algún beneficio.

Al respecto, un joven comunitario nos dice que:

Hablando del bosque... hay bastantes riquezas, hay cerros, hay ríos, hay árboles frutales, y hay árboles listos, hay aves, mamíferos. También allí están los árboles de un gran valor, y hay un sin número de piedras, que están dentro del bosque, y todos desde el más pequeño hasta el más grande es de donde vivimos.

Existen similitudes en lo expresado por el grupo y el joven, en ambas citas es notable la visión holística e integradora del ecosistema bosque, proveedor de bienes y servicios, elementos fundamentales para la satisfacción de necesidades básicas. Sin embargo, en la expresión del grupo se percibe el bosque como un elemento clave para garantizar el buen vivir, en cambio el joven lo visualiza como una oportunidad de generar riquezas, lo que pareciere ser una lógica acumulativa que pudo haber sido adquirida de otras culturas, pues como lo plantea el Fondo Indígena (2008), el conocimiento es dinámico y constantemente se agregan nuevos conocimientos.

A pesar de esta dinámica del conocimiento, en el caso de Miguel Bikan, su cosmovisión del mundo les ha permitido la formación de valores y normas, coincidiendo con lo planteado por Danbolt (1994). Por su parte, Ostrom (1990), nos dice que esos valores y normas son la clave para la constitución de instituciones que garantizan la gestión colectiva de los recursos.

En este sentido, una institución que aún guarda mucho respeto en las comunidades miskitu son los ancianos, poseedores de conocimientos, que se transmiten a los más jóvenes. En base a esto, es probable que la visión de acumulación de riqueza se vea limitada por las normas e instituciones establecidas en la comunidad de Miguel Bikan y tal y como lo plantea Deruyttere (2001), su racionalidad económica no es de acumulación sino de relación armónica con el entorno y uso respetuoso de los recursos naturales para el bienestar de toda la comunidad, por lo que la palabra riqueza puede referirse a diversidad biológica y no tanto divisas económicas. 
Los roles y normas creadas, así como la gestión colectiva, relacionada con su racionalidad económica, son factores fundamentales que orientan a la sustentabilidad del bosque.

\section{Espiritualidad en los bosques}

La espiritualidad de la comunidad de Miguel Bikan, se ve fuertemente influenciada por el cristianismo; sin embargo, aún persisten la espiritualidad desde la concepción indígena. De cualquier modo, ambos pensamientos (cristiano o indígena) se basan en la relación respetuosa con las plantas, los animales, la medicina y otros elementos que se pueden obtener del bosque.

Dios creó esta tierra y después, dijo aprovéchenlo con cuidado... si nosotros rezamos a Dios y le pedimos poder cazar un animal no puedes cazar una manada, ya que Dios sólo da a uno lo necesario... si no aprovechamos nuestros recursos con cuidado no vamos a tener nada para el futuro... (Comunitario de Miguel Bikan, 2010)

Desde la cosmovisión indígena el Dios al que se hace mención sería, según Cox (1998), el principal Dios de los miskitus, creador de todo y el que les ha facilitado todo "Wan Aisa". Este Dios, siempre está presente y exige el cumplimiento de las tradiciones, por lo tanto, favorece el cumplimiento de normas locales.

Aún prevalece la espiritualidad desde la concepción indígena, muchas de ellas están relacionadas al castigo, por parte de seres extraordinarios, por el mal uso o abuso de los recursos. Estas historias no son sólo cuentos o leyendas, muchas de ellas son experiencias vividas por algunos de los participantes y con el tiempo se han ido fortaleciendo hasta convertirse en normas de control para el uso responsable de los recursos naturales.

Es importante notar que existe la percepción que se ha roto la armonía entre los recursos naturales y los seres humanos, y esto ha permitido, hasta cierto punto, el uso racional de los recursos naturales.

Mi padre me contaba una historia, antes cuando uno vivía en armonía con los recursos, todo se pedía con permiso y en estos tiempos no... Se pedía permiso al bosque o al río cuando uno iba a pescar. Y te digo que si uno vuelve a tomar esos hábitos ancestrales no tendría esos problemas que se ven ahorita.

La espiritualidad practicada en la comunidad y que se hace objetivamente con el respeto al bosque y sus componentes, el uso de lo necesario para la familia, consumo responsable, ha derivado en normas de control que se traduce positivamente en tradiciones, es la clave para el uso sustentable del bosque. 


\section{Acciones de aprovechamiento}

Además de las acciones cotidianas de subsistencia diaria relacionada a las prácticas agrícolas, cacería y pesca, también se realizan actividades de aprovechamiento forestal de beneficio familiar y colectivo.

Por un lado, el aprovechamiento familiar se realiza en las parcelas productivas asignadas a cada familia dentro de la comunidad, y por otro, el aprovechamiento llevado a cabo en las áreas de reserva de bosque comunal, manejadas colectivamente y que se encuentran alejadas del poblado comunal. Aquí es donde se realizan los planes generales de manejo (PGM) y/o planes de aprovechamiento forestal (PAF) con que cuenta la comunidad.

En el caso del aprovechamiento familiar, cada familia posee dentro del territorio comunal un área asignada de trabajo que consta de 5-10 hectáreas. Parte del sitio lo destinan como área agrícola (1-2 hectáreas), en donde cultivan granos básicos, principalmente (arroz, frijol) y tubérculos. El resto del área está destinada principalmente para colectar plantas medicinales, recoger frutas silvestres, leña, cacería y aprovechamiento de madera a pequeña escala. Cada una de estas actividades cotidianas conlleva a un uso sustentable del bosque.

No obstante, más del 8o\% de la población se dedica principalmente a la agricultura como actividad económica, la cual está destinada principalmente al autoconsumo y un poco para la comercialización en los mercados locales de Waspam y Bilwi. El enfoque es de racionalidad económica.

Al consultar a un anciano de la comunidad sobre el uso del bosque en el pasado nos dijo:

...antes no había mucho interés por los árboles, y nuestra forma de pensar sólo estaba en la siembra, por eso había mucha comida.... se recolectaba la resina de los árboles en bidones, en galones, en botellitas para almacenarla y al fin del año cada quien la vendía. No había ningún proyecto, no había ninguna ayuda de afuera, y nadie aguantaba hambre... no nos metíamos mucho con los árboles, a veces para nuestras casas... .

Lo mencionado por el anciano refleja su forma de vida de los años 6o, 70 del siglo pasado, en donde su sistema se basaba en un ambiente comunal y el bosque les proveía de lo necesario para sobrevivir. Parecía ser un sistema de manejo del bosque sustentable, ya que les proveí de lo necesario para el sostenimiento de la familia. 
Cabe mencionar que para esa época el aprovechamiento del bosque estaba en manos de algunas trasnacionales que poseían equipos de extracción (STANDART, NIPCO), y la negociación era con el Estado. Este último había definido las tierras estatales y tierras comunales. En este sentido a la comunidad de Miguel Bikan se le asignó un área de 15,000 hectáreas, a través de un título de propiedad otorgado por el IAN.

Entonces, los comunitarios no tenían participación activa en el aprovechamiento de madera y se dedicaban a la agricultura, caza y pesca, y a la extracción de productos no maderables del bosque (resina de pino). Obviamente, que después del retiro de las trasnacionales (década de los ochenta) y apertura de libre comercio (década de los noventa), el interés por la madera a nivel nacional se incrementó, así como los madereros libres, entonces se presentaron a las comunidades e incitaron la venta de madera.

El aprovechamiento familiar de madera se limita a esta área asignada, sólo en los casos en que la parcela tenga árboles aprovechables. Inicia con la autorización del líder comunal para la extracción de hasta 500 pies tablares por año. Esta práctica se hace con el propósito de que las familias se apoyen monetariamente y puedan resolver algunas necesidades inmediatas, principalmente la obtención de herramientas de trabajo.

Así lo explica un líder de la comunidad:

Lo que se hace en el bosque son dos cosas, cada familia tiene su parcela donde realiza su siembra y si en tu parcela tienes árboles y tienes una necesidad puedes aprovechar un máximo de 500 pt y debes de pagar un tronconaje a la autoridades comunales y lo otro es el plan (PGM) y el PAF...

Es importante resaltar que el consumo de leña representa una de las principales formas de aprovechamiento del bosque, pues la mayoría de los comunitarios la utilizan como fuente de energía para la preparación de alimentos. Esta madera es recolectada del bosque de pinares principalmente. Un estudio realizado por el IREMADES-URACCAN, estima que anualmente se aprovechan unos $780 \mathrm{~m}^{3}$ en todo el sector de Tasba Raya (URACCAN-IREMADES-, 2009).

En síntesis, la experiencia de aprovechamiento familiar presenta elementos útiles de trabajo conjunto y colectivo, que podrían ser de mucha importancia para desarrollar un programa de sostenibilidad de uso de los recursos forestales, con la participación activa de la comunidad. No obstante, la extracción a pequeña escala pero sumatoria podría ser de gran impacto en el bosque.

Por otro lado, el desconocer las tasas de regeneración natural, composición de especies, sobrevivencia, así como las tasas de conversión de la cobertura vegetal para otros fines y porcentaje de tierras forestales con cambios ecológicos fundamentales, 
podría orientar a daños futuros en el bosque de gran magnitud, principalmente en la biodiversidad del ecosistema.

En otro orden, el trabajo colectivo está directamente relacionado a la protección de su territorio y al aprovechamiento del bosque en beneficio de la comunidad. El aprovechamiento se hace en las áreas comunales manejadas colectivamente y destinadas para dicho uso.

Antes de los años ochenta, no se daba ningún aprovechamiento en el área comunal, el área de bosque era más que todo para la cacería y recolección de plantas medicinales. Se estima que el aprovechamiento forestal comunal se inicia en el 2000, cuando el INAFOR dio el primer permiso de aprovechamiento forestal, por $283 \mathrm{~m}^{3}$.

La decisión de hacer algún aprovechamiento se toma en la asamblea comunal, de igual manera se define el objetivo por el cual se va a aprovechar. Un informante de la comunidad agrega:

hacemos reunión para decidir sobre la madera que vamos a sacar, ... nosotros aserramos madera en la comunidad para apoyar a los ancianos, a las mujeres viudas y enfermas y para reparar las escuelas y las iglesias, también cuando queremos comprar un piano o planta eléctrica para la iglesia...

Lo externado por el comunitario, tiene que ver con la decisión del uso de los recursos colectivos y la distribución de los mismos. Es un proceso consultado en asamblea comunal y es del saber en la comunidad.

Es así que en los últimos 10 años (2000-2010), la comunidad ha aprovechado 500 hectáreas de bosque en distintos sitios del territorio y sin ningún plan de manejo, pero acompañado de permisos de aprovechamiento comunal extendidos por el INAFOR.

Poschen (2000:5), nos explica que la pobreza que resulta de una distribución extremadamente desigual de la riqueza, de la falta de acceso o de control sobre los recursos, particularmente la tierra y de la carencia de alternativas para una subsistencia basada en la agricultura marginal, es probablemente el factor más dinámico para la destrucción de los bosques.

En este enfoque de aprovechamiento colectivo, los líderes comunales ofrecen a madereros la oportunidad de extraer determinada cantidad de madera, con la condición de que se contrate toda la mano de obra de la comunidad y el pago de tronconaje. Al final lo que le queda a la comunidad es únicamente empleo y el pago del tronconaje (impuesto pagado a la comunidad por volumen aserrado). El tronconaje obtenido por la madera vendida $\left(100 \mathrm{US} / \mathrm{m}^{3}\right)$, es para resolver alguna necesidad comunal y distribuida entre ancianos, viudas, entre otros. 
Uno de los principales problemas planteados por la comunidad es que la distribución social es inequitativa, sólo algunos se benefician de las regalías de la comercialización de la madera; entonces, pareciera ser que no a todos les interesa la protección y conservación del bosque orientada a la sostenibilidad y sustentabilidad. Mientras que los beneficios ambientales son considerados como externalidades sin costos para los comunitarios (agua limpia, aire, fauna del bosque, pesca de agua dulce,..).

La visión comunitaria es que el gobierno nacional, a través del INAFOR, es el que tiene que enfrentar los gastos directos para apoyar el manejo sostenible. El manejo forestal sostenible constituye un proceso que involucra orientar el bosque en varias direcciones que permita sobre todo asegurar la calidad de vida y el sostenimiento de las capacidades productivas.

No obstante, Ostrom (1990) sostiene que los poseedores de un recurso común tienen la capacidad de establecer instituciones por ellos mismos, para controlar las formas de uso y acceso de ese recurso o recursos que comparten colectivamente. También dice que al establecer reglas y diseñar acuerdos entendidos y aceptados por todos, es posible fomentar estrategias de cooperación para mantener los recursos de uso común, ya sean agua, bosques, animales de caza o pesca, pastizales, etc., y lograr un aprovechamiento más eficiente de los mismos.

\section{Normativas locales de uso y manejo del bosque}

A diferencia de lo planteado por Hardin (1968), sobre el acceso abierto a los recursos naturales, las comunidades indígenas tienen definido el uso y manejo de sus recursos naturales, donde no todos los individuos tiene derecho sobre la propiedad y el uso de los recursos.

Las normas de uso son esenciales para la conservación de los recursos, algunas de estas normas como la zonificación de usos de áreas, limitan ciertas actividades que se pueden realizar. Un ejemplo de lo anterior es lo planteado por uno de los participantes de los grupos focales.

...nosotros en el manejo de bosque lo hacemos con cuidado, lo usamos pedazo por pedazo. Cada familia tiene un área donde realiza su agricultura y solo ellos hacen uso de esa área, pero también tenemos áreas comunales que todos podemos usar, pero con el permiso de las autoridades comunales.

Una cosa importante de rescatar es el hecho de que no tienen definida la cantidad de área destinada por familia para realizar la agricultura. En el caso de Miguel Bikan, podría ser que ha habido una revitalización de las costumbres ancestrales, sin que esto signifique que no exista un control por parte de las autoridades comunales, quienes vigilan que no se haga un uso inadecuado o excesivo de los recursos. 
En cuanto al aprovechamiento forestal, existen normas que rigen la cantidad de producto que puedes aprovechar, esta norma es aplicable principalmente en el área comunal; sin embargo, en el área familiar, la cantidad de madera que puedes aprovechar va estar en dependencia de las posibilidades económica de cada familia, así como las reglas establecidas por el INAFOR.

\section{Como bien señala don Tobías comunitario de Miguel Bikan}

$\mathrm{Si}$ alguna persona (comprador externo) tiene interés en cierta cantidad de madera, lo que hacemos es que nos repartimos entre varios y nos ponemos de acuerdo cuanta madera puede sacar cada uno según su capacidad. Por lo general anda entre 300 y 400 pies tablares por familia. No todos aprovechan la madera, pero lo que lo hacen deben de pagar un porcentaje a las autoridades y así todos nos beneficiamos.

El caso de la cacería, se menciona que anteriormente era muy flexible, sin embargo, con el embate del huracán Félix, han notado que la cantidad de animales ha disminuido lo que ha llevado a que los cazadores se limiten en la cantidad de animales que pueden cazar y respetando los animales en gestación y las crías, de manera que garanticen la perpetuidad y recuperación de los animales.

....La cacería no se controlaba, cada cazador mataba lo que necesitaba, pues había muchos animales, pero después del huracán Félix, existe una preocupación por los pocos animales que se ven, así que no se deben de matar los animales pequeños y embarazados para que puedan recuperarse nuevamente.

Otras normas están regida por creencias que limitan la participación de algunos miembro de la comunidad o el tiempo en el que pueden ser utilizados o aprovechados ciertos recursos.

Cuando las mujeres están reglando no pueden sembrar ni trabajar en las parcelas, pues esto hace que los cultivos no nazcan o que se quemen los cultivos que ya están grandes... Si un hombre tiene embarazada a su mujer, no debe de ir a cazar o pescar por que los animales se corren.

.... la madera para construir casas cuando se corta en luna nueva se empieza a podrir, pero si se corta en luna nueva todo está bien. Lo mismo para la siembra, se entiende que la luna nueva hace crecer las cosas, pero lo que son la yuca y el quequisque se siembran en luna llena, el plátano cuando se siembra en cualquier luna se madura muy rápido, pero cuando se siembra sin luna a su punto.

Todas estas normas de uso, que definen quién, dónde y cuándo se utiliza un determinado recurso está bajo la responsabilidad de las instituciones comunales. 
El síndico es el encargado de velar por los recursos naturales y los asuntos de tierra, pero si viene una empresa o un proyecto para la comunidad, el síndico no puede tomar decisiones solo, ahí tienen que estar los ancianos, el juez, los coordinadores, el síndico, con todos los adultos de la comunidad con los niños, si esto se acepta entre todos, allí se debe de empezar a trabajar sino, no, ese es el plan que se tiene.

Los conflictos relacionados con la gestión ambiental y territorial se relacionan tanto con conflictos internos entre los comunitarios, los líderes e inter comunitarios. En el caso de Miguel Bikan, los principales problemas que mencionan son entre comunidades vecinas que según ellos están usurpando parte del territorio que les pertenece.

Una de las comunidades con las que tienen problema limítrofe es Tasba Pain, sin embargo, el problema más grande desde su percepción es el caso del Polo Lakia Sirpi y Kapri. Estas dos comunidades se encuentran dentro del territorio de Miguel Bikan, pero el título de propiedad ya les fue entregado y en él se menciona a estas comunidades como parte del bloque Wanki Twi - Tasba Raya, han optado por buscar solución a esto. Una de esas soluciones que ellos plantearon en los grupos focales es:

Tal vez no podamos sacar a Kapri y Polo de nuestras tierras, pero pensamos ir a darles un pedazo de tierra definido a ellos y decirles de aquí ya no pueden avanzar ni para atrás ni para adelante. Y esa idea de darles ese pedazo de tierra es de toda la comunidad... Esta gente no tiene ningún convenio con nosotros, son gente de Francia Sirpi y ellos tienen sus tierras en Francia Sirpi, y si fuera legal lo pondríamos en saneamiento pero a como dice la ley indígena no pueden estar en saneamiento.

A la par de delimitarles un área para que permanezca ahí, también han definido estrategias que les garanticen que no seguirán avanzando

... los líderes de la comunidad iremos a definir áreas, alrededor del pedazo que les daremos (a Kapri y Polo) para que vivan los jóvenes de Miguel Bikan que están formando su familia... no importa si ellos (Kapri y Polo) ya habían sembrado, ya que ellos han abusado y Miguel Bikan no puede solo, y somos bastantes y no hay tierras, entonces vamos a ir a cuadrar esas tierras, y en donde ya de aquí no se muevan más para adentro.

Estas estrategias planteadas por la comunidad para solucionar los problemas limítrofes, viene a abonar al desarrollo del buen vivir, pues están consciente que los habitantes de Kapri y Polo, también son miskitus y por ende, no pueden estar en problema con su propia gente. Lo importante de esto es que, aun cuando no esté escrito, ya han iniciado un proceso clave para el saneamiento, el cual es la elaboración de normas de convivencia con los terceros dentro de su comunidad. Esta misma 
experiencia, puede ser retomada por otras comunidades del mismo territorio para solucionar el problema actual de invasión por parte de colonos mestizos, garantizando el bien común, la paz y armonía.

\section{Conclusión}

- Se percibe, por parte de los comunitarios, que los jóvenes han adquirido una visión más mercantilista que pone en riesgo la sostenibilidad de los recursos y la socavación del sistema colectivo. Sin embargo, esta visión de los jóvenes con la adecuada incorporación del conocimiento ancestral de los mayores podría abonar de manera positiva al manejo forestal sostenible, puesto que el mercado es importante para la sostenibilidad.

- El bosque es percibido, parte de los comunitarios de manera holística e integradora, donde no se limita a simple productos maderables, sino que incluye productos no maderables, servicios que se pueden obtener, así como al ser humano mismo.

- El bosque es aprovechado de dos maneras en la comunidad, por un lado el aprovechamiento familiar, el cual se realiza dentro de la parcela productiva de cada familia y el aprovechamiento comunal, el cual se realiza en el área de reserva comunal, a través del PGMF y el PAF. .

- La cosmovisión de los comunitarios, ha permitido la formulación de normas y reglas que garantizan de una u otra forma, la sustentabilidad del bosque; sin embargo, las nuevas generaciones han ido irrespetando algunas de estas normas.

\section{Lista de referencias}

Cox, A. (1998). Cosmovisión de los pueblos de Tulu Walpa: según relatos de los sabios ancianos miskitos. Ed. Carlos Alemán. Managua: URACCAN.

Danbolt, D. (1997). Encuentro de cosmovisón. El encuentro entre la cultura y la religión de los autóctonos del Cañar y el Evangelio. Quito. Ediciones Abya-Yala.

Deruyttere, A. (2001). Pueblos indígenas, desarrollo con identidad: algunas reflexiones de estrategias.

Fondo Indígena. (2008). Modulo espiritualidad, conocimientos e historia de los pueblos indígenas de Abya Yala. La Paz, Bolivia. 
Hardin, G. (1968). The tragedy of the commons. Science, Vol. 162, pp. 1243-1248. La presente es una versión revisada de "La tragedia de los espacios colectivos", en H. E. Daly (ed.), Economía, ecología y ética: ensayos hacia una economía en estado estacionario, 1992, México, Fondo de Cultura Económica. pp. 111-124.

INAFOR. (2009). Resultados del inventario nacional forestal: Nicaragua 2007-2008. 2 ed. Managua. Autor. 232 pp.

López, G. (2008a). Plan general de manejo forestal de la comunidad de Miguel Bikan. PANA PANA

López, G. (2008b). Aprovechamiento y Manejo Forestal Comunitario en Bosques Afectados por el Huracán Félix en la comunidad de Miguel Bikan, Waspam, río Coco. PANA PANA-Fundación FORD

Ostrom, E (1990). Governing The Commons. The Evolution of Institutions for Collective Action. Cambridge, Uniersity Press. Primera edición en español en el 2000, traducido por Corina Iturbide Calvo y Adriana Sandoval con el título "El gobierno de los bienes comunes. La evolución de las Instituciones Colectivas", Editado por la Universidad Nacional Autónoma de México y el Fondo de Cultura Económica, México

PANA PANA. (2009). Sistematización del proyecto piloto para la validación de técnicas de salvamento, extracción y transformación de árboles caídos por efecto del huracán Félix en tres comunidades indígenas de la RAAN. El autor.

Poschen, P. (2000). Criterio e indicadores sociales para el manejo forestal sostenible. GTZ

Pocop, R. (2008). La madre naturaleza desde la cosmovisión Maya. En territorios y recursos naturales: Saqueo versus el buen vivir.

URACCAN-IREMADES. (2009). Línea base sobre la administración de los recursos forestales en el territorio de Tasba Raya. Documento inédito. 